\title{
Technè
}

La science au service de l'histoire de l'art et de la préservation des biens culturels

41 | 2015

Arts textiles antiques et modernes. Approche scientifique et restauration

\section{Étude de colorants d'une offrande textile (Y16) exceptionnelle, découverte sur le site de Cahuachi (culture Nasca)}

Study of the dyes used in a rare textile offering (Y16) discovered on the site of Cahuachi (Nazca civilization)

Nathalie Boucherie, Witold Nowik et Dominique Cardon

\section{OpenEdition} Journals

Édition électronique

URL : http://journals.openedition.org/techne/4443

DOI : $10.4000 /$ techne.4443

ISSN : 2534-5168

\section{Éditeur}

C2RMF

\section{Édition imprimée}

Date de publication : 25 novembre 2015

Pagination : 46-54

ISBN : 978-2-7118-6248-1

ISSN : $1254-7867$

Référence électronique

Nathalie Boucherie, Witold Nowik et Dominique Cardon, «Étude de colorants d'une offrande textile (Y16) exceptionnelle, découverte sur le site de Cahuachi (culture Nasca)», Technè [En ligne], 41 | 2015, mis en ligne le 08 août 2020, consulté le 11 mars 2021. URL : http://journals.openedition.org/techne/ 4443 ; DOI : https://doi.org/10.4000/techne.4443

\section{(c) (i) (9)}

La revue Technè. La science au service de l'histoire de l'art et de la préservation des biens culturels est mise à disposition selon les termes de la Licence Creative Commons Attribution - Pas d'Utilisation Commerciale - Pas de Modification 4.0 International. 


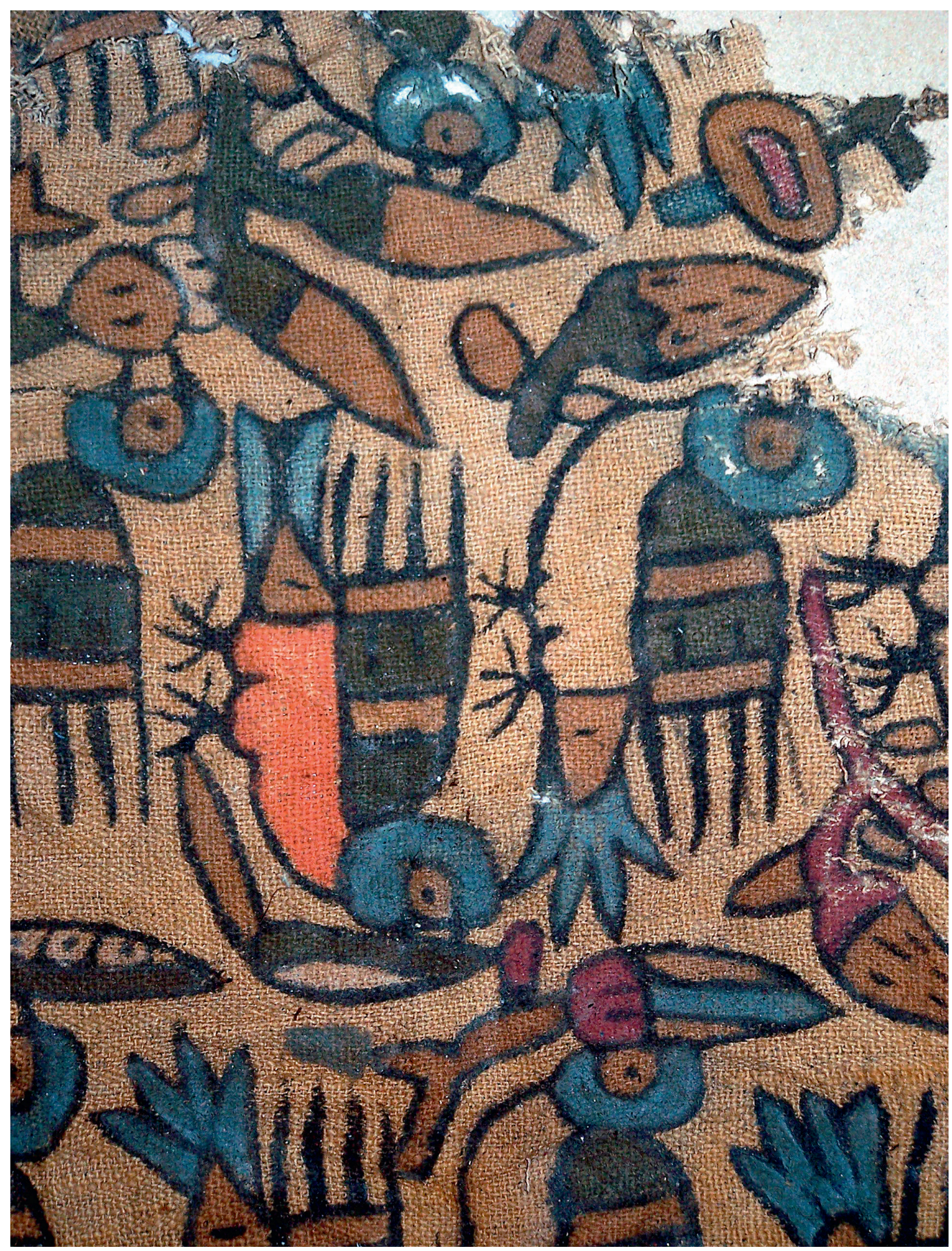

Fig. 1. Oiseaux peint sur la robe 45

(détail du décor).

(c) Nathalie Boucherie. 
Nathalie Boucherie Witold Nowik Dominique Cardon

\section{Étude de colorants d'une offrande textile (Y16) exceptionnelle, découverte sur le site de Cahuachi (culture Nasca)}

Study of the dyes used in a rare textile offering (Y16) discovered on the site of Cahuachi (Nazca civilization)

\begin{abstract}
Résumé. En 1998, une offrande textile exceptionnelle a été découverte sur le site majeur de la culture Nasca : Cahuachi. Soixante-trois ballots textiles y avaient été enterrés ensemble. Ces paquets renfermaient principalement des vêtements cérémoniels, dont les lisières étaient très souvent ornées d'éléments de décor confectionnés avec des fils teints de plusieurs couleurs. Vingt et un objets textiles ont été sélectionnés afin de mener une étude approfondie sur les colorants employés en teinture pour réaliser les décors polychromes de ces textiles somptueux. Elle met en lumière un choix limité de colorants préparés à partir de sources végétales : le bleu des Indigofera, le rouge des Galium et le jaune, probablement de l'espèce Salix humboldtiana, ainsi que leurs mélanges binaires. Quelques colorants particuliers, comme le violet d'indigo, le noir contenant de l'acide ellagique ou un jaune à flavonoüdes provenant d'une source végétale non identifiée, ont aussi été mis en évidence.
\end{abstract}

Mots-clés : Nasca, Pérou, offrande textile, identification, fibres, colorants.
Abstract. In 1998, an extraordinary textile offering was found in Cahuachi, an important centre of the Nazca civilization. Sixty-three bundles of textiles had been buried together on this site. The bundles contained mainly ceremonial clothes, the edges of which were very often decorated with patterns made of threads of various colours. Twenty-one textile items were selected in order to carry out an in-depth study of the dyes used in the colourful decoration on these sumptuous garments. The results showed that a limited number of plant dyes was employed: blue from Indigofera, red from Galium, and yellow, probably issued from the Salix humboldtiana, as well as their binary mixtures. Some particular dyes, namely an indigo violet, a black containing ellagic acid, and a flavonoid yellow obtained from an unidentified plant, were also detected. dyes.

Keywords. Nazca, Peru, textile offering, identification, fibres,

\section{Introduction}

La culture Nasca est une ancienne civilisation préhispanique qui est apparue sur la côte sud du Pérou vers 200 avant J.-C. et qui s'est éteinte vers 700 après J.-C. Grâce aux conditions désertiques de la région, de nombreux textiles se sont relativement bien conservés. Une partie des textiles Nasca est issue de fouilles clandestines, mais un nombre important a aussi été mis au jour lors des campagnes de fouille de plusieurs sites, notamment Cahuachi, le centre politico-cérémoniel majeur pendant la phase Nasca ancien (50 avant J.-C. -350/400 après J.-C.). Ainsi, en 1998, les archéologues du site de Cahuachi ont découvert un puits creusé dans les anciennes structures architecturales du site ${ }^{1}$. Ce puits correspond à une intrusion effectuée durant la dernière phase d'occupation de Cahuachi, qui se situe aux alentours de 350-400 après J.-C. Deux grands ballots textiles en furent extraits. Chacun d'eux contenait des petits paquets textiles qui correspondaient à un ou plusieurs tissus ou vêtements soigneusement pliés : au total, soixante-trois petits paquets furent mis au jour.

Ce dépôt semble correspondre à une offrande rituelle, mais il est très probable que le puits était une tombe à l'origine et peut-être les vêtements découverts appartenaient-ils au défunt enterré dans ce lieu².

Sur une période de deux ans, les paquets textiles ont été ouverts un à un et étudiés à plusieurs reprises ${ }^{3}$.

Il s'agit d'une découverte majeure : elle constitue à elle seule un corpus textile conséquent. Ces nombreux vêtements richement décorés, dont le contexte est connu, offrent la possibilité d'étudier le style, l'iconographie, les thèmes, la typologie des vêtements et les techniques développées par les artisans textiles Nasca. Les pièces textiles se trouvent dans l'ensemble en état de conservation assez bon, mais plusieurs vêtements montrent des lacunes importantes. D'autres tissus, très fragiles, se réduisent en poudre dès une simple manipulation.

Nathalie Boucherie, archéologue, chercheur indépendant (listrao@hotmail.com). Witold Nowik, chimiste, ingénieur de recherche, département Recherche, C2RMF (witold.nowik@ culture.gouv.fr). Dominique Cardon, historienne des techniques, directeur de recherche émérite, CNRS/CIHAM UMR 5648 (cardon.dominique@wanadoo.fr) 
Le style des textiles de l'offrande correspond à la phase Nasca 3 selon l'évaluation de Mary Frame ${ }^{4}$. D'après son analyse, les tissus correspondent presque exclusivement à des vêtements de femme ${ }^{5}$ avec quarante robes de types différents, ainsi que huit châles. Toutefois, pour Maria Bastiand, certains types de robes correspondent à des vêtements masculins ${ }^{6}$.

La couleur prédominante du corpus demeure le bleu. Quelques vêtements sont simples, sans décor ni couleur. En revanche, plusieurs robes sont teintes d'une, deux ou trois couleurs et comportent des décors peints ou fabriqués avec des plumes ; parfois, elles présentent même les deux types de décoration à la fois. Souvent aussi, des galons brodés ou des figures tridimensionnelles multicolores ornent les lisières : ces vêtements constituent des pièces de choix pour étudier les décors colorés. Le corpus offre donc une formidable occasion d'étudier les traditions tinctoriales de cette culture par rapport à celles des Paracas, mieux documentées ${ }^{7}$. D'après les vestiges textiles retrouvés, il semble que Paracas, la culture précédant Nasca, ait une production textile assez restreinte en couleurs, mais qui s'est enrichie au fil du temps. À la fin de la période Paracas, alors qu'émerge la culture Nasca, apparaît une tradition tinctoriale plus riche, notamment avec les textiles Paracas Necrópolis et Nasca ancien, à peu près contemporains. La présente étude des textiles Nasca a donc été entreprise dans le but de compléter nos connaissances sur l'histoire de la teinture dans le Pérou ancien.

\section{Les objets textiles étudiés}

Dans le cadre d'une thèse de doctorat sur les matières colorantes des textiles $\mathrm{Nasca}^{8}$, vingt-trois objets textiles de l'offrande ont fait l'objet de prélèvements afin d'étudier de manière approfondie ces matières colorantes.

L'analyse concerne seize robes et jupes, trois châles et quatre autres tissus dont la fonction est différente (tableau I). Parmi les robes, deux sont uniquement teintes, cinq présentent un décor peint (fig. 1), six un décor de plumes et deux sont à la fois ornées d'un motif peint et de plumes. Les châles sont tous décorés de motifs peints et deux d'entre eux sont également ornés de plumes (fig. 2). Parmi les quatre tissus, deux sont teints, un présente un décor de carreaux blanc et marron et le dernier est décoré de petits végétaux brodés sur toute la surface (fig. 3).

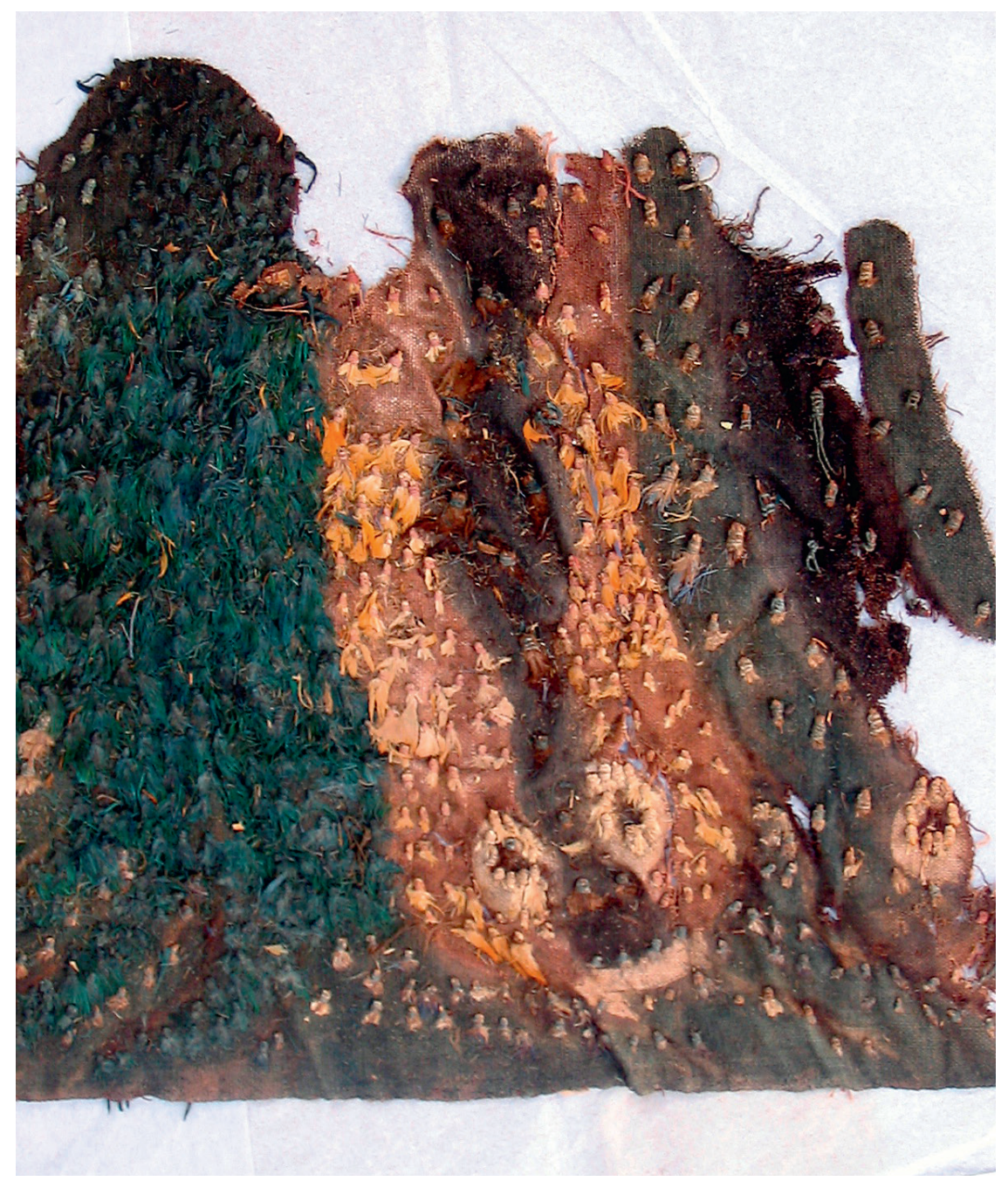

Fig. 2. Motif serpentiforme peint et recouvert de plumes de la jupe 10 (détail du décor). (C) Nathalie Boucherie. 


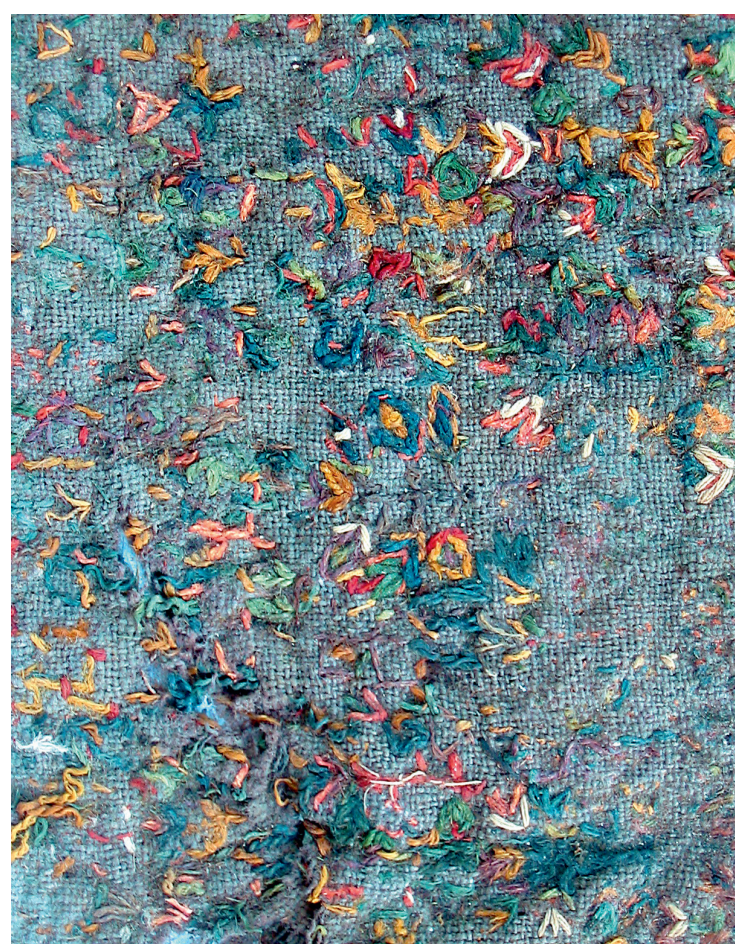

Fig. 3. Végétaux brodés sur le tissu 57 (détail du décor). (c) Nathalie Boucherie.

\section{Les techniques textiles}

L'armure des lés qui constituent tous les vêtements analysés est invariablement l'armure toile. Nous n'avons pas observé d'autres techniques de tissage.

Des éléments de décoration ont souvent été cousus le long des lisières. Il s'agit généralement de galons tissés en armure toile et parfois en tapisserie, brodés et bordés de franges bouclées, ou de petites figures tridimensionnelles réalisées minutieusement à l'aiguille selon la technique du réseau-broderie au point bouclée ${ }^{10}$, caractéristique de la production Nasca ${ }^{11}$.

On trouve aussi des bordures décorées de languettes. Elles sont réalisées soit au point bouclé simple (autrement désigné point de tulle) sur un élément de base, généralement un galon brodé qui était ensuite cousu, soit coupées directement dans le tissu et renforcées par des points de couture.

\section{Les fibres textiles}

Les pièces du corpus ont été tissées entièrement avec des fibres de coton et les éléments de décor ont été réalisés principalement avec des poils de Camélidés.

\section{Tableau I. Typologie des objets textiles étudiés}

\begin{tabular}{|c|c|}
\hline $\begin{array}{l}\text { Type d'objet }{ }^{9} \\
\text { et } \mathbf{n}^{\circ} \text { de référence }\end{array}$ & Description \\
\hline Châle 34 & Vêtement peint en bleu et en marron et garni de plumes \\
\hline Châle 37 & Des oiseaux ont été peints sur la surface et une frise tridimensionnelle décore les lisières \\
\hline Châle $61 \mathrm{~B} / \mathrm{C}$ & $\begin{array}{l}\text { Châle léger en toile peu serrée de coton. } 61 \mathrm{~B} \text { : toile bleue peinte de motifs noirs. } 61 \mathrm{C} \text { : toile bleue garnie de plumes } \\
\text { jaunes. Galon tubulaire réalisé au point bouclé }\end{array}$ \\
\hline Jupe 10 & $\begin{array}{l}\text { Toile peinte de grands motifs (un visage flanqué de serpent) et décorée de plumes (jaune orangé, blanches, bleues, } \\
\text { marron) }\end{array}$ \\
\hline Jupe 12 & Toile peinte de motifs serpentiformes de dimension moyenne, noirs, rouges, marron et blancs \\
\hline Jupe 18 & $\begin{array}{l}\text { Toile rose décorée du motif répété d'un serpent à deux têtes, peint en noir avec quelques rehauts de peinture pourpre. } \\
\text { Présence de franges carrées découpées dans la toile }\end{array}$ \\
\hline Robe 02 & $\begin{array}{l}\text { Robe complète, bleue, recouverte de plumes bleues, marron, noires et blanches. Ces dernières dessinent un collier } \\
\text { blanc sur le corsage. Galons brodés sur la lisière du rabat et de la jupe }\end{array}$ \\
\hline Robe 04 & Robe teinte en bleu, à panneau central, sans décor ajouté \\
\hline Robe 09D & Robe bleue et ocre, à panneau central, avec des plumes jaunes sur le corsage \\
\hline Robe 09E & Vêtement d'enfant, bleu et ocre, décoré de plumes. Présence d'aplats de peinture noire délimitant des zones de tissu \\
\hline Robe 11 & Vêtement en toile bleu foncé, en très mauvais état \\
\hline Robe 13 & Corsage bleu foncé et jupe (très fragmentaire) peinte d'un motif répété de l'Être mythique anthropomorphe \\
\hline Robe 20 & $\begin{array}{l}\text { Robe à panneau central, bleu clair et bleu foncé, marron et blanc, recouverte de plumes. Les bords inférieurs du rabat } \\
\text { et de la jupe sont ornés d'un galon en toile rouge brodé d'oiseaux }\end{array}$ \\
\hline Robe 23 & $\begin{array}{l}\text { Robe avec un corsage bleu foncé à noir et une jupe décorée de motifs peints d'oiseaux en train de dévorer } \\
\text { des petits animaux }\end{array}$ \\
\hline Robe 27 & $\begin{array}{l}\text { Robe à panneau central, en toile de coton crème/marron clair. Le panneau est constitué de deux lés : l'un bleu, } \\
\text { décoré de plumes bleues, l'autre marron avec un décor peint en damier, recouvert de plumes jaunes et noires }\end{array}$ \\
\hline Robe 28 & Robe ayant subi des remaniements, avec un corsage bleu-vert et une jupe crème, recouverte de plumes \\
\hline Robe 36 & Robe bleue recouverte de plumes \\
\hline Robe 45 & Robe avec un corsage bleu et une jupe entièrement peinte d'oiseaux en train de se nourrir \\
\hline Textile 08 & Deux fragments d'une robe : l'un bleu et bleu foncé, l'autre marron clair \\
\hline Tissu 17E & Délicate toile ouverte, crêpée, marron \\
\hline Tissu 49 & Toile ouverte crêpée, teinte en rose \\
\hline Tissu 57 & $\begin{array}{l}\text { Bande de toile en coton marron, entièrement brodée, et différents éléments de décor (galon tissé en tapisserie, } \\
\text { bande tubulaire) }\end{array}$ \\
\hline Tissu 61A & Toile de coton bleu et crème avec des fils de chaîne discontinus \\
\hline
\end{tabular}


Par conséquent, la principale fibre textile utilisée pour les tissus de cette offrande reste le coton. Sa fibre est généralement de couleur blanc crème, mais elle peut aussi présenter différents tons naturels de marron ${ }^{12}$. Certaines robes sont en effet composées d'un ou plusieurs tissus en fibres de coton naturellement marron. C'est aussi en jouant avec ces différents tons que des motifs à carreaux ont pu être réalisés.

L'identification précise de l'espèce de Camélidés à partir de l'observation sous microscopie optique des fibres archéologiques reste encore difficile en raison du manque de pertinence des référentiels utilisés. En effet, on emploie couramment des poils de Camélidés actuels comme éléments de comparaison. Or, nous savons, grâce aux recherches de Jane Wheeler et de ses collègues ${ }^{13}$, qu'une hybridation intense entre lama et alpaga commença à partir de la conquête espagnole : ainsi, à l'époque préhispanique, les stratégies d'élevage sélectif ont permis d'obtenir des toisons de qualité (fine, unie, abondante, homogène), mais l'abandon de ces pratiques a rendu les toisons actuelles hétérogènes et plus grossières. De fait, les poils de Camélidés domestiques actuels ne sont pas comparables à ceux des alpagas et lamas préhispaniques.

L'étude des fibres montre l'absence presque totale de fibres très grossières $(63 \mu \mathrm{m})$ : elles ont été observées seulement dans trois échantillons et cela de manière pratiquement résiduelle $(<2 \%)$. Leur rareté peut être liée soit à un triage manuel de la laine effectué par les artisans Nasca avant le filage, soit à la bonne qualité de la laine (homogène et fine) des races laineuses utilisées.

Par ailleurs, plusieurs échantillons présentent un diamètre moyen relativement petit $(16 \mu \mathrm{m})$ et se composent principalement de fibres fines ( $90 \%$ ), avec de rares fibres grossières, voire aucune : ces caractéristiques les rapprochent des toisons des Camélidés sauvages (vigogne et guanaco). L'absence quasi totale de fibre grossière pourrait alors être due à un éjarrage manuel, c'est-à-dire à l'élimination des poils longs et raides que l'on appelle des jarres ${ }^{14}$.

En revanche, les échantillons présentant un diamètre moyen plus grand ( $23 \mu \mathrm{m}$ ) et un pourcentage en fibres moyennes beaucoup plus important ( $60 \%$ ) pourraient correspondre vraisemblablement à des poils de Camélidés domestiques (lama et alpaga).

Tableau II. Classification des résultats obtenus selon la nature des fibres et des colorants

(Les composés du tableau II sont annotés de la façon suivante: Ell : acide élagique ; Flav : flavonö̈de; Ind : indigotine ; Inr : indirubine ; Lut : lutéoline; Pur: purpurine.)

\begin{tabular}{|c|c|c|c|}
\hline Fibres & Couleur & $\begin{array}{l}\text { Principaux } \\
\text { composés } \\
\text { colorants }\end{array}$ & Objet \\
\hline coton & bleu & Ind & Châle 34 \\
\hline coton & bleu & Ind & Châle $61 \mathrm{~B} / \mathrm{C}$ \\
\hline coton & bleu & Ind & Jupe 10 \\
\hline coton & bleu & Ind & Textile 08 \\
\hline coton & bleu & Ind & Robe 04 \\
\hline coton & bleu & Ind & Robe 09E \\
\hline coton & bleu & Ind & Robe 11 \\
\hline coton & bleu & Ind & Robe 13 \\
\hline coton & bleu & Ind & Robe 23 \\
\hline coton & bleu & Ind & Robe 28 \\
\hline coton & bleu & Ind & Robe 36 \\
\hline coton & bleu & Ind & Robe 45 \\
\hline coton & bleu & Ind & Tissu 61A \\
\hline coton & rose & Pur & Tissu 49 \\
\hline poils de Camélidés & bleu & Ind & Châle 37C \\
\hline poils de Camélidés & bleu & Ind & Robe 20 \\
\hline poils de Camélidés & bleu & Ind & Robe 27 \\
\hline poils de Camélidés & bleu & Ind & Tissu 57 \\
\hline poils de Camélidés & vert mordoré & Ind + Flav & Robe 20 \\
\hline poils de Camélidés & vert & Ind + Lut & Châle 37C \\
\hline poils de Camélidés & vert & Ind + Lut & Châle 61B/C \\
\hline poils de Camélidés & vert & Ind + Lut & Jupe 18 \\
\hline poils de Camélidés & vert & Ind + Lut & Robe 02 \\
\hline poils de Camélidés & vert & Ind + Lut & Tissu 57 \\
\hline poils de Camélidés & noir & Ell & Robe 02 \\
\hline poils de Camélidés & noir & Ind + Pur & Châle $61 \mathrm{~B} / \mathrm{C}$ \\
\hline poils de Camélidés & noir-brun & Ind + Pur & Robe 20 \\
\hline poils de Camélidés & violet & Ind + Pur & Châle 37C \\
\hline poils de Camélidés & violet-noir & Ind + Pur & Robe 28 \\
\hline poils de Camélidés & violet & Ind + Pur & Robe 27 \\
\hline poils de Camélidés & violet & Inr & Châle 34 \\
\hline poils de Camélidés & mauve & Inr & Robe 20 \\
\hline poils de Camélidés & violet & Inr & Robe 20 \\
\hline poils de Camélidés & violet & Inr & Tissu 57 \\
\hline poils de Camélidés & jaune & Lut & Châle 37C \\
\hline poils de Camélidés & jaune & Lut & Châle 61B/C \\
\hline poils de Camélidés & jaune & Lut & Robe 02 \\
\hline poils de Camélidés & jaune & Lut & Robe 27 \\
\hline poils de Camélidés & jaune & Lut & Tissu 57 \\
\hline poils de Camélidés & jaune orangé & Lut & Tissu 57 \\
\hline poils de Camélidés & orange & Pur & Châle 37C \\
\hline poils de Camélidés & orange & Pur & Tissu 57 \\
\hline poils de Camélidés & rose & Pur & Châle 37C \\
\hline poils de Camélidés & rouge & Pur & Châle 34 \\
\hline poils de Camélidés & rouge & Pur & Châle 37C \\
\hline poils de Camélidés & rouge & Pur & Châle 61B/C \\
\hline poils de Camélidés & rouge & Pur & Robe 02 \\
\hline poils de Camélidés & rouge & Pur & Robe 20 \\
\hline poils de Camélidés & rouge & Pur & Robe 27 \\
\hline poils de Camélidés & rouge & Pur & Robe 28 \\
\hline poils de Camélidés & rouge & Pur & Tissu 57 \\
\hline
\end{tabular}




\section{Les colorants}

L'étude des colorants a été réalisée par chromatographie en phase liquide ${ }^{15}$ avec détection en spectroscopie UV-Vis (abbr. ang. HPLC-PDA). Ces analyses ont été menées sur vingt et un textiles de l'offrande Y16 (quatorze robes, trois châles, quatre tissus).

\section{Trois principales sources de teinture}

Les analyses ont permis de mettre en évidence l'utilisation de plusieurs colorants (tableau II), appartenant principalement à trois types de sources.

Le premier type est déterminé par l'utilisation d'indigo, un colorant caractérisé par la présence dominante d'indigotine (Ind). Une ou plusieurs espèces d'indigotiers (Indigofera spp.), qui constituent des sources d'indigo au Pérou, ont peut-être été utilisées. En effet, parmi les restes botaniques découverts à Cahuachi, Indigofera suffruticosa Mill. a été identifié $^{16}$. Ce type de colorant donne des teintes bleues.

Le deuxième type de colorant se caractérise par la prédominance de purpurine (Pur) et par plusieurs autres anthraquinones en plus faible concentration. Parmi ces molécules secondaires, l'alizarine est parfois présente. Cette composition est propre aux espèces de Galium du Pérou, dont les racines sont traditionnellement utilisées en teinture ${ }^{17}$. Par ailleurs, d'après les recherches récentes, la présence d'alizarine caractérise seulement quelques espèces de Galium $^{18}$. Les colorants alors obtenus donnent des teintes rouges.

La composition du troisième groupe est caractérisée par la prédominance de lutéoline (Lut), associée à l'apigénine, au chrisoériol, ainsi qu'à d'autres flavones en plus faible concentration. Nous n'avons pas trouvé de concordance exacte parmi nos références de teinture préparées à partir de plusieurs sources locales connues ${ }^{19}$, mais celle qui s'en rapproche le plus a été obtenue avec les feuilles d'un saule répandu en Amérique centrale et en Amérique du Sud et présent autour du site de Cahuachi : Salix humboldtiana Willd. Toutefois, on ne peut écarter l'emploi d'autres sources colorantes non répertoriées. Les colorants de ce type sont jaunes.

Les sources tinctoriales pouvaient être utilisées seules ou en combinaisons diverses. Par combinaison de deux colorants extraits des plantes citées ci-dessus, les teinturiers obtenaient des violets et des noirs purs ou presque (bleu + rouge), ainsi que des verts (bleu + jaune).

\section{Les teintures sur coton}

Au vu des résultats présentés dans le tableau II, on remarque que si le coton est teint, c'est presque exclusivement avec de l'indigo (Ind), un colorant de cuve polyvalent, et un des rares qui soit adapté aux fibres cellulosiques. La seule exception pour le coton concerne le tissu 49 qui a été teint en rose, une coloration obtenue avec un colorant à base de purpurine (Pur) issu des Galium. Le colorant des Galium appartient à la classe des colorants à mordant, qui sont destinés à la teinture des fibres protéiques (laine, poils, soie). La teinture des fibres cellulosiques (coton, lin) avec les colorants à mordant est assez complexe et implique souvent l'utilisation de tanins. Les tanins n'ont pas été détectés dans cet échantillon, ce qui a pu rendre le processus de coloration peu efficace. En effet, l'échantillon prélevé était de couleur rose pâle, ce qui peut être expliqué par une difficulté de faire «monter » le colorant sur les fibres ou par la faible tenue de la teinture dans le temps. Les deux effets résultent logiquement d'une fixation inefficace du colorant.

\section{Les teintures sur laine}

L'indigo (Ind) constitue également le colorant bleu des fils en poils de Camélidés.

Les teintures binaires associant le colorant d'indigo au colorant jaune à flavonoïdes ont produit les verts des fils en poils de Camélidés. En prenant en compte les composés minoritaires du colorant à base de lutéoline, il semble que cette composition corresponde à notre référence de teinture obtenue avec Salix humboldtiana ${ }^{20}$, arbre présent dans l'environnement des fouilles ${ }^{21}$. Un seul résultat fait exception : en effet, un fil vert, prélevé sur la robe 20, a été teint avec une plante à indigo et une autre plante à flavonoïdes (Flav). La source de ces flavonoïdes n'a pas été identifiée faute de référence correspondante.

Les rares noirs observés ont été obtenus de deux manières. Un premier type de noir a été identifié sur la robe 02. Ici, les fibres de Camélidés ont été teintes avec un tanin ellagique (Ell), probablement mordancées avec un sel de fer. Les tanins ellagiques sont assez répandus dans le règne végétal, mais la comparaison du résultat d'analyse avec nos références semble indiquer qu'ils pourraient être issus du tara (Caesalpinia spinosa Kuntze $)^{22}$, un arbre local. Deux autres exemples de noir, présents dans le châle $61 \mathrm{~B} / \mathrm{C}$ et dans la robe 20 , ont été teints par combinaison d'une teinture rouge extraite des Galium, riches en purpurine (Pur), et d'un bleu d'indigo (Ind). La coloration foncée est le résultat d'une grande accumulation de ces deux colorants, qui, en concentration plus faible, ont donné certains violets, parfois assez soutenus, comme pour la robe 28. Les colorations violette et mauve étaient également obtenues par une teinture de cuve, avec les extraits d'Indigofera comme pour le bleu d'indigo, mais dans ce cas, le bain de teinture était très riche en indirubine (Inr), un isomère rouge de l'indigotine. L'indirubine est généralement un composé fortement minoritaire dans l'indigo, néanmoins la production d'indirubine peut être favorisée en fonction du $\mathrm{pH}$ et de la température du bain ${ }^{23}$. La présence simultanée de l'indirubine (Inr) rouge et de l'indigotine (Ind) bleue dans le bain permet d'obtenir des nuances de violet en fonction de leur proportion respective. Il est intéressant d'observer que les violets à base d'indirubine ont été utilisés ici uniquement pour les fils en poils de Camélidés, alors qu'il est possible d'employer ce colorant sur le coton. 
La coloration jaune des fils a été obtenue avec un colorant contenant de la lutéoline (Lut), probablement Salix humboldtiana, et les orange et rouges avec un colorant contenant de la purpurine (Pur), issu des Galium.

\section{Discussion}

Afin de mieux comprendre pour quelle raison un colorant a été choisi plutôt qu'un autre en vue d'obtenir la même couleur, les résultats ont été considérés en fonction des objets. Dans le tableau III, nous avons présenté uniquement les objets comportant plusieurs couleurs caractérisées par l'analyse chromatographique. Les objets ayant des fils teints uniquement d'une seule couleur (en majorité du coton teint en bleu) n'ont pas été inclus dans cette comparaison.

On remarque ainsi que, dans le châle 34 , coexistent un fil bleu teint à l'indigo et un fil rouge teint avec un colorant à base de purpurine. Pourtant, le violet analysé correspond à une teinture d'indigo avec prédominance d'indirubine et non à un mélange Ind/Pur, alors que les deux ingrédients étaient disponibles. Cette remarque s'applique aussi au tissu 57, ainsi qu'à la robe 20. Néanmoins, dans ce dernier exemple, nous trouvons également une teinture avec bi-composants Ind/Pur, mais cette fois-ci pour produire du noir. Cette observation permet de dire que la production de violets d'indigo coexistait avec la production de teintures composées bleu/rouge, qui peuvent aussi donner des tons violets. La teinture composée violette a aussi été identifiée sur un fil du châle 37C.

La gamme réduite de colorants (Ind pour le bleu, Lut pour le jaune et Pur pour le rouge), que nous pouvons considérer comme «standard » dans notre étude, est représentée par les vêtements polychromes suivants : châle $37 \mathrm{C}$, châle $61 \mathrm{~B} / \mathrm{C}$, robe 27 et robe 28 .

Cette gamme est complétée par l'usage de violet d'indigo (Inr) pour le châle 34, la robe 20 et le tissu 57, et du colorant Flav pour la composante jaune d'un fil vert prélevé sur la robe 20, ce qui constitue la deuxième particularité de cet objet. Par ailleurs, pour la robe 02 , nous remarquons l'usage d'ellagitanin (Ell) et non du mélange Ind/Pur pour le noir, alors que les deux composants sont utilisés pour le vert (Ind) et pour le rouge (Pur) dans ce même vêtement.

Ces colorants particuliers pourraient caractériser certains artisans qui cherchaient à innover. L'emploi du colorant Flav s'expliquerait comme une expérimentation par les teinturiers avec les différentes sources locales. L'emploi d'ellagitanin (Ell) nécessite en revanche de comprendre l'influence du mordant sur la couleur obtenue. En effet, les tanins ellagiques donnent souvent des roux ou des bruns quand ils sont appliqués avec un mordant achromatique (comme l'aluminium), alors qu'avec un mordançage au fer, ils produisent une teinture noire. Par conséquent, l'obtention de noir à partir de tanins indique une certaine maîtrise de ce procédé de teinture différent de celui fondé sur la superposition d'une teinture à mordant (Pur) sur une teinture de cuve (Ind). Ce dernier cas est pourtant plus fréquent dans notre corpus. De même, la production d'un violet (Inr) dérivé de l'indigo relève d'un savoir-faire précis : obtenir les teintes violettes exige des conditions de teinture particulières avec un $\mathrm{pH}$ et une température expérimentalement maîtrisés.

Il semble ainsi que les teinturiers Nasca savaient produire des mauves avec les plantes à indigo, traditionnellement employées pour teindre en bleu. À ce jour, aucune autre étude de colorants n'a mis en évidence une telle composition sur des textiles archéologiques du Pérou : cette composition pourrait être caractéristique de la production Nasca. Cela dénoterait une expertise particulière dans la manipulation de l'indigo afin d'obtenir les teintes voulues.

Il est possible que ces teintures insolites caractérisent la production textile d'un ou de quelques artisans ou ateliers dédiés à l'élaboration des vêtements somptueux pour l'élite.

En effet, les nombreux textiles de l'offrande Y16 ont manifestement été confectionnés par plusieurs artisans ou ateliers. Par exemple, les décors peints exécutés dans des styles différents indiquent vraisemblablement la participation de divers artisans ${ }^{24}$. De la même manière, les quelques différences remarquées dans le choix des colorants pourraient caractériser un ou plusieurs artisans/ateliers.

Tableau III. Couleurs et colorants des textiles en fonction de l'objet

\begin{tabular}{|c|c|c|c|c|c|c|c|c|c|}
\hline \multirow{2}{*}{ Objet } & \multirow{2}{*}{$\begin{array}{c}\text { Rouge } \\
\text { Pur }\end{array}$} & \multirow{2}{*}{$\begin{array}{c}\text { Jaune } \\
\text { Lut }\end{array}$} & \multicolumn{2}{|c|}{ Vert } & \multirow{2}{*}{$\begin{array}{l}\text { Bleu } \\
\text { Ind }\end{array}$} & \multicolumn{2}{|c|}{ Violet } & \multicolumn{2}{|c|}{ Noir } \\
\hline & & & Ind + Lut & Ind + Flav & & Inr & Ind + Pur & Ind + Pur & Ell \\
\hline Châle 37C & + & + & + & & + & & + & & \\
\hline Châle 61B/C & + & + & + & & + & & & + & \\
\hline Robe 27 & + & + & & & + & & + & & \\
\hline Robe 28 & + & & & & + & & + & & \\
\hline Châle 34 & + & & & & + & + & & & \\
\hline Tissu 57 & + & + & + & & + & + & & & \\
\hline Robe 20 & + & & & + & + & + & & + & \\
\hline Robe 02 & + & + & + & & & & & & + \\
\hline
\end{tabular}


Ainsi, on note que les violets d'indigo ont été identifiés sur les éléments de décor de la robe 20 (fig. 5), du châle 34 (fig. 4) et du tissu 57 (fig. 3).

Malgré l'état très fragmentaire du châle 34 (fig. 4), on remarque qu'il partage des caractéristiques communes avec la robe 20 (fig. 5) : une couleur dominante dans les tons bleus (bleu-vert pour le châle), ponctuée de brun (et de blanc pour la robe), un décor de plumes sur presque toute la surface et un galon aux tons prédominants rouges et violets. Il semble qu'un oiseau, les ailes semi-dépliées, ait été peint sur le châle ${ }^{25}$. La disposition des restes de plumes bleues, blanches et brunes, et le dessin d'un fil blanc cousu sur la robe semblent représenter le plumage d'un oiseau avec les flancs et les ailes bleus, le cou bleu et blanc, la poitrine brune et blanche et la queue marron.

Tous ces éléments ainsi que l'identification des violets d'indigo sur ces deux seuls vêtements amènent à supposer qu'il pourrait s'agir d'un ensemble assorti, certainement confectionné ou du moins décoré par le même artisan. Grâce à l'étude du style des décors peints, Mary Frame a remarqué que certains châles et robes présentaient des motifs peints très similaires et devaient constituer un ensemble assorti ${ }^{26}$. De même, dans notre étude, on pourrait dire que le «style tinctorial » original d'un artisan ou d'un atelier pourrait permettre de reconnaître les pièces d'un même costume.

Il est possible, enfin, que le tissu 57, pour lequel un autre violet d'indirubine a été identifié, ait été produit par le même artisan/atelier que celui qui a élaboré les décors de la robe 20 et du châle 34 . Ou bien peutêtre appartenait-il à un autre artisan. Mais dans les deux cas, la recette permettant d'obtenir des violets d'indigo était pratiquée par un petit nombre d'artisans ou d'ateliers.

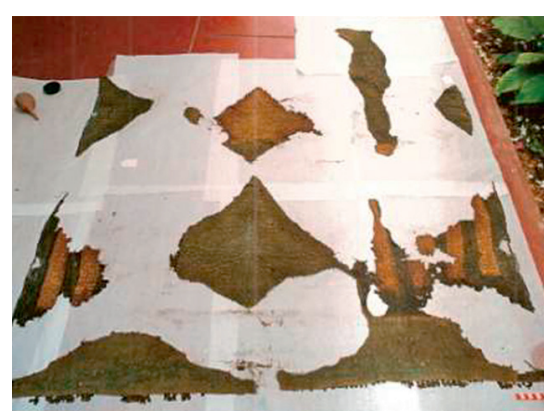

Fig. 5. Robe 20. (C) Maria Bastiand.

\section{Conclusion}

L'étude de ce corpus textile exceptionnel révèle l'existence de teinturiers spécialisés qui ont atteint un haut niveau de savoir-faire. C'est grâce à la connaissance de l'environnement et des techniques héritées de leurs ancêtres que les teinturiers Nasca ont su produire un nombre de plus en plus varié de couleurs et de nuances avec seulement quelques sources colorantes et sont ainsi passés maîtres dans l'art de la teinture. Aux époques antérieures, les populations de la côte sud produisaient des tissus moins colorés. C'est au début de l'ère Nasca que la recherche de polychromie se trouve à son apogée, comme en témoignent les textiles présentés ici. Cette recherche de couleurs et nuances variées a amené les artisans Nasca, tout en conservant et pratiquant les procédés traditionnels, à poursuivre les expérimentations et à innover pour étendre la gamme de couleurs. C'est ainsi que notre étude a mis en évidence la fabrication de teintures violettes à partir d'une source d'indigo. Ces teintures constituent un exemple de recherche technique exceptionnel et elles caractérisent la production tinctoriale Nasca. Dans l'ensemble, on remarque une certaine homogénéisation concernant le choix des matières colorantes, mais chaque artisan conservait sa liberté dans l'élaboration des recettes, ce qui explique les quelques exceptions observées. Les maîtres teinturiers Nasca constituaient donc une main d'œuvre qualifiée et spécialisée, avec un savoir-faire maîtrisé et des recettes propres. 
Notes

1. Olano et Llanos, 1999.

2. Llanos, 2009, p. 260.

3. De Concini, 2002, Frame, 2005,

Bastiand, 2010.

4. Frame, 2005 , note 15 .

5. Frame, 2005.

6. Bastiand, 2010, p. 117 .

7. Wouters, Rosario-Chirinos, 1992 ,

Saltzman, 1978, Saltzman et al., 1963.

8. Boucherie, 2014.

9. Frame, 2005

10. Le terme « réseau-broderie au point bouclé » est de Raoul d'Harcourt

(D'Harcourt, 1948 et 2008, p. 106-108).

Cette technique est aussi désignée comme

" réseau bouclé ressemblant au tricot " (Desrosiers, 2008).

11. O’Neale, 1934, Sawyer, 1997,

p. 136-159, Nunez-Regueiro et al., 2006.

12. Vreeland, 1999.

13. Wheeler et al., 1992 et 1995.

14. Jarre : "Poil de couverture des mammifères, long et raide, de densité faible, dépassant les poils de bourre ou duvet, mais moins rigide que les épines. (Les crins sont des poils de jarre.) " Définition proposée par le dictionnaire Larousse.

15. Analyses effectuées au Laboratoire de recherche des Monuments historiques.

16. Piacenza, 2002.

17. Dutra Moresi, 1999, Dutra Moresi et Wouters, 1997, Fester et Lexow, 1943, p. 233-240.

18. Devia et Cardale, 2011

19. Boucherie et Nowik, à paraître.

20. Boucherie et Nowik, à paraître.

21. Piacenza, 2002

22. Boucherie et Nowik, à paraître.

23. Ushida et Kawasaki, 2003, Ushida,

2005, Kohama et al., 2005.

24. Frame, 2005.

25. Frame, 2005

26. Frame, 2005 , p. 29

\section{Références bibliographiques}

Boucherie N., Nowik W., à paraître, "The dyestuffs in the Nasca Culture (Peru): The construction of an analytical database", Dyes in History and Archaeology, vol. 26 (Actes du colloque, Vienne, 2007), Jo Kirby ed., Archetype Publications, London.

Desrosiers S., 2008, « Les techniques de Paracas-Necrópolis : techniques et décors », dans Exp. Paris, 2008, Paracas, Trésors inédits du Pérou ancien [Exposition, musée du quai Branly, 2008], Flammarion, p. 52-57.

Devia B., Cardale de Schrimp M., 2011, "Color y técnica: nuevos avances en el estudio de textiles precolombinos del Sur de Colombia y el Norte del Ecuador", dans Victoria Solanilla Demestre (dir.), VJornadas
Internacionales de Textiles Precolombinos,

Actes du Colloque (Barcelone,

29 novembre-2 décembre 2010).

Grup d'Estudis Precolombins,

Barcelone, p. 45-61.

Dutra Moresi C. M., Wouters J., 1996, "HPLC Analysis of extracts, dyeings and lakes prepared with 21 species of Relbunium", Dyes in History and Archaeology, vol. 15 (Actes du colloque, Manchester, 1996), Archetype Publications, London, p. 85-97.

Fester G. A., Lexow S. G., 1943, "Las raíces del genero 'Relbunium' en la tintorería americana”, Anales de la Sociedad Cientifica Argentina, vol. 136, p. 233-240.

Frame M., 2005, "What the women were wearing: a deposit of early Nasca Dresses and shawls from Cahuachi, Peru, The Textile Museum Journal, 2003-2004, vol. 42 et 43, Washington, p. $13-53$.

Harcourt (d') R., 1948, « Un tapis brodé de Paracas, Pérou ", Journal de la Société des Américanistes 37, p. 241-257.

Harcourt (d') R., 2008, [1934], Les Textiles anciens du Pérou et leurs techniques, Flammarion, musée du quai Branly, Paris.

Kohama Y., Ushida S., Ueno T., Tanimitsu K., 2005, "Production of indirubine under acidic conditions and the application to dyeing with fresh leaves of indigo plants", Journal of Home Economics of Japan, vol. 56, $\mathrm{n}^{\circ} 12$, p. 879-888 (en japonais).

Llanos D., 2009, Le bassin du Rio Grande de Nazca, Pérou. Archéologie d'un état andin, 200 avant J.-C.-650 ans après J.-C., BAR International Series 1990, Archaeopress, Oxford.

Núñez-Regueiro P., Desrosiers S., Deliens J., Oger B., Nowik W., 2006,

"Bordure de textile nasca ancien ", Technè, 23, p. 107-110.

O’Neale L., 1934, "Peruvian needleknitting”, American Anthropologist, N. S., vol. 36, n 3 , p. 405-430.

Piacenza L., 2002, "Evidencias botánicas en asentamientos Nasca”, Boletín del Museo de Arqueología y Antropología, 5 (1), Universidad Nacional Mayor de San Marcos, UNMSM, Lima, p. 3-13.

Saltzman M., 1978, “The identification of dyes in archaeological and ethnographic textiles", in Carter, G. F., Archaeological chemistry II, Washington D.C., American Chemical Society, Advances in Chemistry, p. 172-185.

Saltzman M., Keay A. M., Christensen J., 1963, "The identification of colorants in ancient textiles", Dyestuffs, vol. 44, $\mathrm{n}^{\circ} 8$, p. 241-251.

Ushida S., 2005, "Principle of indigo production and indigo dyeing", dans Proceedings of the International Symposium on Indigo Dyeing Culture, Jui-Tsung Lee ed., Taipei, p. 19-28.
Ushida S., Kawasaki M., 2003, "Dyeing a purple shade using fresh leaves of the Japanese Indigo Plant", Dyes in History and Archaeology, vol. 19, Archetype Publications, London, p. 106-108.

Sawyer A., 1997, Early Nasca Needlework, Lawrence King and Alan Marcuson eds., Primitive Arts Ltd., London.

Vreeland J., 1999, « Du coton naturellement coloré ", Pour la Science, n 266, p. 62-68.

Wheeler J. C., Russel A. J. F., Stanley H. F., 1992, "A measure of loss: prehispanic llama and alpaca breeds", Archivos de Zootecnia, vol. 41, n 154 (extra), p. 467-475.

Wheeler J. C., Russel A. J. F., Redden H., 1995, "Llamas and Alpacas:

Pre-conquest Breeds and Post-conquest Hybrids", Journal of Archaeological Science, 22, p. 833-840.

Wouters J., Rosario-Chirinos N., 1992, "Dye analysis of pre-Columbian Peruvian textiles with highperformance liquid chromatography and diode-array detection", Journal of the American Institute for Conservation, vol. 31, $\mathrm{n}^{\circ} 2$, article 7, p. 237-255.

\section{Documents inédits}

Bastiand M., 2010, Los textiles rituales de Nasca en Cahuachi, thèse de doctorat, Universidad Nacional Mayor de San Marcos, Lima.

Boucherie N., 2014, La couleur dans la civilisation Nasca : production tinctoriale et picturale, thèse de doctorat, Université Lumière Lyon 2, Lyon.

De Concini E. A., 2002, Le pitture "alate" dei Nasca. Contributo allo studio stilisticoiconografico dei tessuti precolombini, Tesi di Laurea, Università Ca' Foscari di Venezia.

Dutra Moresi C. M., 1999, Avaliação quimica do potencial de especies natives de Relbunium (garança americana) como fornecedoras de corantes e pigmentos e sua aplição ao estudo de obras de arte, thèse de doctorat, Universidade Federal de Minas Gerais, Belo Horizonte, Brésil.

Olano J., Llanos D., 1999, "Informe de excavación Cahuachi 1998, sector Y16, Experimento 67", in Proyecto Nasca, Informe final de la campaña 1998, Orefici G., Pieri E., Sanchez A. eds, CISRAP, Brescia, presentado al INC de Lima, p. $94-130$. 